\title{
Cinematic rendering for ischiofemoral impingement syndrome caused by osteochondroma: a case description
}

\author{
Jian Liu ${ }^{1,2 \#}$, Xinge Cheng ${ }^{1,2 \#}$, Chong Tian ${ }^{2}$, Rongpin Wang ${ }^{2}$, Jiaxiang Chen $^{3}$, Xiaoyong Zhang ${ }^{2}$, \\ Xianchun Zeng ${ }^{2}$ \\ ${ }^{1}$ Department of Graduate School, Zunyi Medical University, Zunyi, China; ${ }^{2}$ Department of Radiology, Guizhou Provincial People’s Hospital, Key \\ Laboratory of Intelligent Medical Imaging Analysis and Accurate Diagnosis of Guizhou Province, International Exemplary Cooperation Base of \\ Precision Imaging for Diagnosis and Treatment, Guiyang, China; ${ }^{3}$ Guizhou University School of Medicine, Guiyang, China
}

"These authors contributed equally to this work.

Correspondence to: Xianchun Zeng. Department of Radiology, Guizhou Provincial People's Hospital, 83 Zhongshan East Rd, Guiyang 550002, China. Email: zengxianchun04@foxmail.com.

Submitted May 15, 2021. Accepted for publication Aug 31, 2021.

doi: 10.21037/qims-21-525

View this article at: https://dx.doi.org/10.21037/qims-21-525

\section{Introduction}

In the fields of diagnosis and treatment, full knowledge and accurate understanding of detailed anatomical information is a vital requirement. Cinematic rendering (CR) is an emerging methodology for three-dimensional visualization of volumetric imaging. The use of a global lighting model and direct and indirect lighting is the most prominent feature of CR to create images (1). CR images contain more shadowing and depth details that are not available from maximum intensity projection and volume rendering (2). Therefore, CR may promote image interpretation and improve the visualization of anatomical features such as tissues and bones.

Ischiofemoral impingement syndrome (IFIS), first described in 1977, is defined as a narrowing of the space between the ischial tuberosity and lesser trochanter, resulting in entrapment of the quadratus femoris (QF) (3). IFIS is still considered a rare pathology (4). Osteochondroma is one of the most common benign tumors (5), but osteochondroma of the lesser trochanter of the femur has rarely been reported. Here, we report a 54-year-old male with IFIS secondary to osteochondroma of the lesser trochanter and the use of CR.

\section{Case presentation}

A 54-year-old man presented with a 1-year history of worsening severe pain in the right hip joint, without a history of lower extremity trauma or surgery. The pain intensity increased during walking. Physical examination demonstrated a full range of motion of the hip joints without atrophy, swelling or deformity. The patient had received conservative treatment in other hospitals but did not show any response to the treatment.

To clarify the etiology, the patient received related examinations in the radiology department. At admission, pelvic X-ray and computed tomography (CT) showed an osseous prominence protruding from the lesser trochanter, narrowing the distance between the lesser trochanter and the ischial tuberosity (Figure $1 A, 1 B$ ). The bone marrow of the osteochondroma that compressed the QF was continuous with the bone marrow of the lesser trochanter. In this report, the CR image from CT data could realistically show the relationship between the parent bone and the osteochondroma from different views (Figure 2). Magnetic resonance imaging (MRI) revealed that the osteochondroma (size: $22 \times 11 \times 34 \mathrm{~mm}^{3}$ ) originated from the lesser trochanter and the hyperintense signal was indicative of edema in the QF (Figure 3A). The MRI signals revealed severe narrowing of the right ischiofemoral space (IFS) and the quadratus femoris space (QFS) (distances of 10 and $6 \mathrm{~mm}$, respectively) compared with the contralateral side. In addition, the CR image from MRI data had even 

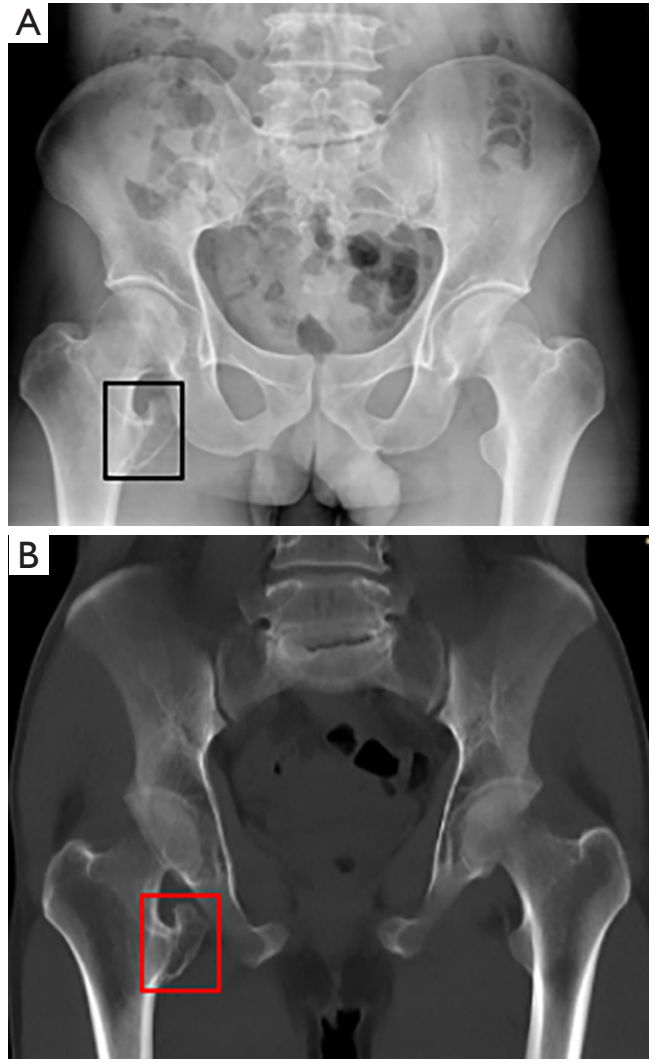

Figure $1 \mathrm{X}$-ray and CT MIP reconstruction results. (A,B) Pelvic X-ray (A) and coronal CT MIP reconstruction (B) (slice thickness: $10 \mathrm{~mm}$ ) show the presence of an osteochondroma of the lesser trochanter and narrowing of the distance between the lesser trochanter and the ischial tuberosity (black and red frames). CT, computed tomography; MIP, maximum intensity projection.

better performance in showing the narrowing of the IFS and edema of the QF (Figure 3B)

The large-sized osteochondroma was surgically removed and was post-surgically confirmed to be an osseous prominence from the lesser trochanter with entrapment of the QF that presented with edema. Histopathological examination showed an osteocartilaginous mass with no cellular atypia or malignant differentiation. Therefore, the diagnosis of osteochondroma was confirmed. Postoperatively, the patient was allowed to use crutches to bear weight and was discharged 4 days later. During the 3-week follow-up, the patient's mobility was dramatically improved, and he was able to walk without crutches.

Ethical approval was granted from the hospital's ethics committee, and all procedures performed in studies involving human participants were in accordance with

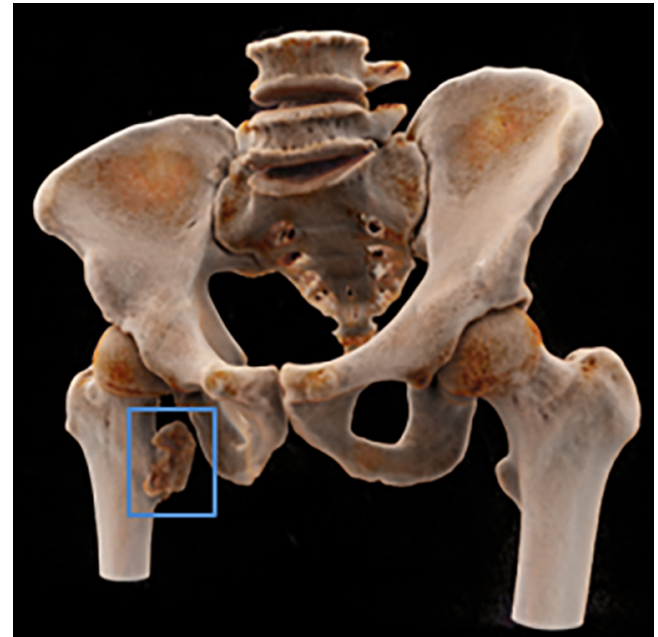

Figure 2 CR image from the CT data at the coronal plane. This CR image contained detailed anatomical information of the osteochondroma in the right lesser trochanter bone and the relationship between the osteochondroma and the parent bone (blue frame). CR, cinematic rendering; CT, computed tomography.
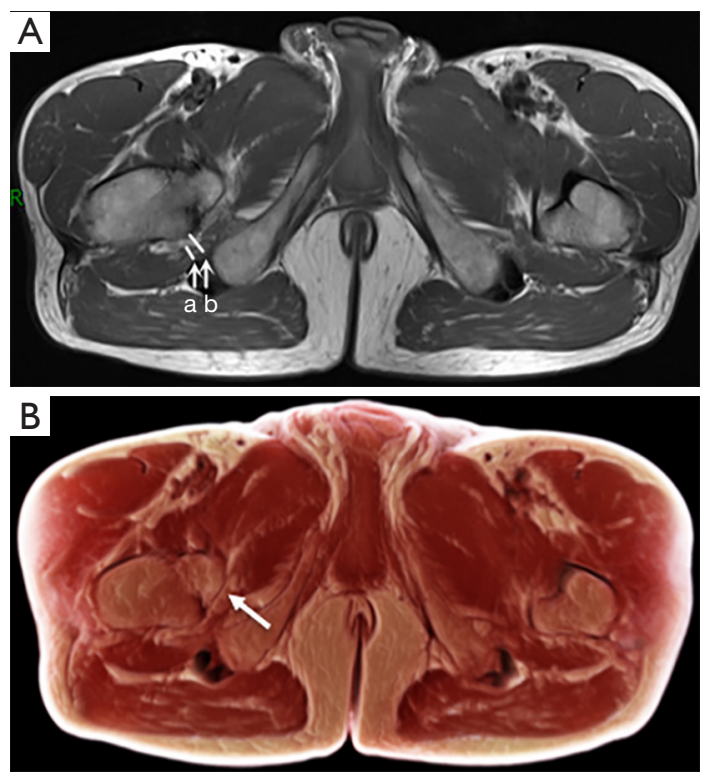

Figure 3 Axial MRI image and CR image from the MRI data. (A) Axial MRI T1-weighted image showing mild edema and focal fatty infiltration in the QFS, and narrowing of the QFS (a, $6 \mathrm{~mm})$ and IFS (b, $10 \mathrm{~mm}$ ). (B) CR image from MRI data, which more realistically demonstrated the narrowing of the IFS and edema of the QF (white arrow), allowing for easier understanding of this disease by the orthopedic surgeon and the patient. MRI, magnetic resonance imaging; CR, cinematic rendering; QFS, quadratus femoris space; IFS, ischiofemoral space; QF, quadratus femoris. 
the ethical standards of the institutional and/or national research committee(s) and with the Helsinki Declaration (as revised in 2013). Written informed consent was obtained from the patient for publication of this case report and accompanying images. A copy of the written consent is available for review by the editorial office of this journal.

\section{Discussion}

Hip impingement is a bone-related condition that can cause hip and pelvic pain (6). The abnormal contact between the acetabulum and the femur or between the ischium and the femur are the two main causes of hip impingement (7). IFIS has not been studied in much detail but has recently received increased recognition $(8,9)$. IFIS is characterized by narrowing the space between the proximal femur and the ischium and compression of the $\mathrm{QF}$, which causes chronic pain in the buttock (10). IFIS with cartilaginous exostosis at the lesser trochanter has been reported (11), but there are few reports of IFIS caused by solitary osteochondroma.

The causes of IFIS can be congenital or acquired. Examples of congenital IFIS include congenital disabilities of proximal femurs, such as valgus deformity of the hips or prominence of the lesser trochanter $(3,7)$. Acquired distortions can arise from post-traumatic changes, osteochondroma originating from the lesser trochanter, intertrochanteric fractures involving the lesser trochanter, valgus-producing intertrochanteric osteotomy, and osteoarthritis $(12,13)$. Osteochondroma is a common benign bone tumor that usually occurs in long tubular bones such as the distal femur, proximal tibia and humerus (14), but rarely occurs in the lesser trochanter of the femur. Osteochondromas are painless bumps, but symptoms such as pain may appear with the growth of the tumor that compresses blood vessels and nerves. In the case reported here, QF edema was observed due to the pressure of the giant osteochondroma on the lesser trochanter. The abnormalities of the QF can cause buttock, hip and groin pain, probably due to the irritation of the sciatic nerve next to the muscle.

For diagnostic purposes, an X-ray of IFIS revealed a reduced distance between the osseous prominences of the lesser trochanter and the ischium and an osteochondroma on the lesser trochanter. When radiographs showed normal findings, the diagnosis of IFIS was confirmed by MRI of the pelvis, which showed narrowing of the IFS or QFS and edema of the QF. The IFS is usually defined as the minimum distance between the lateral cortex of the ischial tuberosity and the medial cortex of the lesser trochanter, while a distance less than $17 \mathrm{~mm}$ indicates an abnormal finding. The QFS is the minimum space with passage of the QF between the superolateral surface of the hamstring tendons and the posteromedial surface of the distal iliopsoas tendon, and QFS less than $8 \mathrm{~mm}$ represents an abnormal finding $(7,15)$. When the QF develops edema, fatty replacement, or atrophy, narrowing of these spaces can occur.

Similarly, narrowing of the IFS and the QFS can compress the QF, further leading to edema and atrophy (16). MRI examination enables precise assessment of the degree of IFS and QFS stenosis and the degree of muscular edema/ fatty replacement and atrophy (12). Radiologists should be aware of the radiographic features of this syndrome.

$\mathrm{CR}$ is a newly developed reconstruction technique for three-dimensional visualization of volumetric imaging data, providing clearer images of vascularized structures and contrast-enhanced blood vessels, tissues, and bones and describing complex anatomical structures more accurately. CR images contain dynamic and continuous information of the lesion, from the surface to the interior. This technique can greatly improve the exchange of information between clinicians and radiologists or between clinicians and patients.

Compared with traditional CT imaging, CR visualization enables surgeons to understand surgical anatomy more accurately and rapidly, independent of the surgeon's level of experience. Therefore, CR can assist general surgeons in preoperative preparation and intraoperative guidance (17).

Lesser trochanter osteochondroma is a rare cause of IFIS, which can limit hip movements and cause severe impingement and QF tear if not diagnosed promptly. For this structural pathology, perhaps the best treatment method is to remove the lesser trochanter osteochondroma surgically.

\section{Conclusions}

Osteochondroma arising from the lesser trochanter is one of the causes of IFIS, the diagnosis of which should be considered when QF edema combined with narrowing of the IFS and QFS is observed. Radiography may be useful for detecting osteochondromas, but cannot evaluate the secondary effect on the soft tissue. MRI is the preferred tool for direct assessment of both narrowings of the IFS and the associated soft tissue edema in the QF. Moreover, CR can provide more photorealistic images to evaluate the 
narrowing of the bony ischiofemoral tunnel and edema or atrophy of the QF. Therefore, the use of multiple approaches for imaging examination can provide a better assessment of IFIS.

\section{Acknowledgments}

Funding: This study was supported by Guizhou Science and Technology Project \{QKHZC[2020]4Y002\}, Guiyang Science and Technology Project \{ZKXM[2020]4-1\}, and Guizhou Science and Technology Department Key Laboratory Project $\{$ QKF[2017]25\}.

\section{Footnote}

Conflicts of Interest: All authors have completed the ICMJE uniform disclosure form (available at https://dx.doi. org/10.21037/qims-21-525). The authors have no conflicts of interest to declare.

Ethical Statement: The authors are accountable for all aspects of the work in ensuring that questions related to the accuracy or integrity of any part of the work are appropriately investigated and resolved. All procedures performed in studies involving human participants were in accordance with the ethical standards of the institutional and/or national research committee(s) and with the Helsinki Declaration (as revised in 2013). Written informed consent was obtained from the patient for publication of this case report and accompanying images. A copy of the written consent is available for review by the editorial office of this journal.

Open Access Statement: This is an Open Access article distributed in accordance with the Creative Commons Attribution-NonCommercial-NoDerivs 4.0 International License (CC BY-NC-ND 4.0), which permits the noncommercial replication and distribution of the article with the strict proviso that no changes or edits are made and the original work is properly cited (including links to both the formal publication through the relevant DOI and the license). See: https://creativecommons.org/licenses/by-nc-nd/4.0/.

\section{References}

1. Yang J, Li K, Deng H, Feng J, Fei Y, Jin Y, Liao C, Li Q. CT cinematic rendering for pelvic primary tumor photorealistic visualization. Quant Imaging Med Surg
2018;8:804-18.

2. Dappa E, Higashigaito K, Fornaro J, Leschka S, Wildermuth S, Alkadhi H. Cinematic rendering an alternative to volume rendering for $3 \mathrm{D}$ computed tomography imaging. Insights Imaging 2016;7:849-56.

3. Johnson KA. Impingement of the lesser trochanter on the ischial ramus after total hip arthroplasty. Report of three cases. J Bone Joint Surg Am 1977;59:268-9.

4. Aguilera-Bohórquez B, Leiva M, Pacheco J, Calvache D, Fernandez M, Cantor E. Pain relief and good functional outcomes after hip endoscopy via posterior approach in patients with ischiofemoral impingement. Knee Surg Sports Traumatol Arthrosc 2021;29:2394-400.

5. Florez B, Mönckeberg J, Castillo G, Beguiristain J. Solitary osteochondroma long-term follow-up. J Pediatr Orthop B 2008;17:91-4.

6. Steppacher SD, Albers CE, Siebenrock KA, Tannast M, Ganz R. Femoroacetabular impingement predisposes to traumatic posterior hip dislocation. Clin Orthop Relat Res 2013;471:1937-43.

7. Torriani M, Souto SC, Thomas BJ, Ouellette H, Bredella MA. Ischiofemoral impingement syndrome: an entity with hip pain and abnormalities of the quadratus femoris muscle. AJR Am J Roentgenol 2009;193:186-90.

8. Ganz R, Slongo T, Turchetto L, Massè A, Whitehead $\mathrm{D}$, Leunig M. The lesser trochanter as a cause of hip impingement: pathophysiology and treatment options. Hip Int 2013;23 Suppl 9:S35-41.

9. Ahn YS, Kim S, Kim WJ, Lim JH, Jung ST. Characteristics of hip impingement syndrome in patients with multiple hereditary exostoses. BMC Musculoskelet Disord 2021;22:153.

10. Yoong P, Mansour R, Teh JL. Multiple hereditary exostoses and ischiofemoral impingement: a case-control study. Skeletal Radiol 2014;43:1225-30.

11. Viala P, Vanel D, Larbi A, Cyteval C, Laredo JD. Bilateral ischiofemoral impingement in a patient with hereditary multiple exostoses. Skeletal Radiol 2012;41:1637-40.

12. Tosun Ö, Çay N, Bozkurt M, Arslan H. Ischiofemoral impingement in an 11-year-old girl. Diagn Interv Radiol 2012;18:571-3.

13. Lerch TD, Zwingelstein S, Schmaranzer F, Boschung A, Hanke MS, Todorski IAS, Steppacher SD, Gerber N, Zeng G, Siebenrock KA, Tannast M. Posterior Extraarticular Ischiofemoral Impingement Can Be Caused by the Lesser and Greater Trochanter in Patients With Increased Femoral Version: Dynamic 3D CT-Based Hip Impingement Simulation of a Modified FABER Test. 
Orthop J Sports Med 2021;9:2325967121990629.

14. Aldashash F, Elraie M. Solitary osteochondroma of the proximal femur causing sciatic nerve compression. Ann Saudi Med 2017;37:166-9.

15. Stenhouse G, Kaiser S, Kelley SP, Stimec J. Ischiofemoral Impingement in Children: Imaging With Clinical Correlation. AJR Am J Roentgenol 2016;206:426-30.

16. Hotait M, Makki A, Sawaya R. Ischiofemoral impingement syndrome: a case report redefining this condition.

Neurosurg Rev 2016;39:707-9.

17. Elshafei M, Binder J, Baecker J, Brunner M, Uder M, Weber GF, Grützmann R, Krautz C. Comparison of Cinematic Rendering and Computed Tomography for Speed and Comprehension of Surgical Anatomy. JAMA Surg 2019;154:738-44.

Cite this article as: Liu J, Cheng X, Tian C, Wang R, Chen J, Zhang X, Zeng X. Cinematic rendering for ischiofemoral impingement syndrome caused by osteochondroma: a case description. Quant Imaging Med Surg 2022;12(2):1647-1651. doi: 10.21037/qims-21-525 